\title{
A new combined Bodian-Luxol technique for staining unmyelinated axons in semithin, resin-embedded peripheral nerves: a comparison with electron microscopy
}

\author{
M. Deprez ${ }^{1}$, C. Ceuterick-de Groote ${ }^{2}$, A. Fumal ${ }^{1}$, M. Reznik ${ }^{1}$, J. J. Martin ${ }^{2}$ \\ ${ }^{1}$ Laboratoire de Neuropathologie, Tour de Pathologie + 1, CHU Université de Liège, B-4000 Sart Tilman, Belgium \\ ${ }^{2}$ Laboratory of Neuropathology, Born Bunge Foundation and University of Antwerp, Antwerp, Belgium
}

\begin{abstract}
Quantitation of unmyelinated fibers (UF) in peripheral nerves has classically relied upon ultrastructural morphometry. Because this method is time-consuming, it is not typically performed in routine analysis of nerve biopsies. We applied the Bodian-Luxol technique to detect unmyelinated axons by light microscopy on semithin sections from resin-embedded nerve tissue. Estimates were compared to ultrastructural counts. The staining appeared highly specific for axons. Excellent correlation was found between optic densities and the population of UF larger than $0.5 \mu \mathrm{m}$. The smallest profiles detected by light microscopy had a diameter close to $0.6 \mu \mathrm{m}$. This new technique is not a substitute for ultrastructural quantitative morphometry of UF, as very small unmyelinated axons, especially regenerating ones, can not be reliably visualized. However, it provides a valuable light microscopic method for evaluating axonal loss among UF.
\end{abstract}

Keywords : Unmyelinated fibers ; Peripheral nerve ; Electron microscopy ; Histochemistry

\section{INTRODUCTION}

In most human cutaneous nerves, $80 \%$ of the axonal population consists of unmyelinated nerve fibers (UF) [12, 21]. Electrophysiological studies have indicated that most unmyelinated axons are derived from dorsal root ganglia and are components of primary afferent units preferentially relaying noxious stimulation. Of these unmyelinated axons $90 \%$ are of the C polymodal nociceptor type and are excited by strong mechanical stimuli, noxious heat, and irritant chemicals [20]. A small subset of UF represents sympathetic efferences that provide innervation to cutaneous appendages $[17,20]$. In any given nerve, vasoconstrictor, sudomotor and sensory nerve fibers are distributed in the same cutaneous area.

The small caliber of unmyelinated axons, ranging from 0.2 to $3 \mu \mathrm{m}$, has always been a limitation to their histological study. Although they were first identified and quantified by light microscopy using silver impregnation techniques [16], their full characterization was achieved with the advent of transmission electronic microscopy. Recognition of UF disease is best achieved by ultrastructural morphometry and relies basically on three parameters: (1) the total number of unmyelinated axons, (2) the number of empty Schwann cell subunits as a gross approximation of the proportion of axons lost, and (3) the size-frequency histogram of unmyelinated axons as an indicator of potential axonal regeneration $[2-5,13-15,18]$.

However, because it is a time consuming and tedious task, ultrastructural quantitation of UF is usually not performed in routine analysis of peripheral nerve biopsy.

Recently, Johnson et al. [7] advocated immunohistochemical identification of axons by light microscopy as a rapid means for estimating UF density. Using the anti-PGP 9.5 antibody, counterstained with Luxol fast blue, they obtained excellent correlation with traditional morphometric estimates. Unfortunately, this method is only suitable for 1- $\mu \mathrm{m}$-thick cross-sections obtained from paraffin-embedded tissue blocks; in our hands, it has often been difficult to match this technical requirement with a good preservation of morphology.

Therefore, we developed a histochemical method for the identification of unmyelinated axons combining the specificity of the Bodian silver impregnation technique with the high resolution of semithin sections from resin- 
embedded nerve tissue. Slides were counterstained with Luxol fast blue and observed under the light microscope. Estimates of the UF population were compared with those obtained by traditional ultrastructural morphometry. This study was aimed at delineating the specificity and sensitivity of this particular method. Absolute numbers of axons were established from isolated fascicles of selected pathological and control cutaneous nerves.

\section{MATERIALS AND METHODS}

\section{Patient selection and nerve processing}

\section{Patient selection}

Control nerves were obtained at autopsy from two Caucasian male patients aged 56 years (control 1) and 51 years (control 2). with no known history of neurological disease. Duration of postmortem autolysis was less than $4 \mathrm{~h}$. Routine examination under optic and electron microscopy failed to show any sign of neuropathy. Pathological nerves were obtained from biopsy samples of two patients with severe neuropathies. Case 1 was a 75-year-old man with chronic axonal sensorimotor polyneuropathy of unknown etiology and case 2 was a 59year-old woman with an axonal form of Guillain-Barré syndrome (GBS) secondary to cytomegalovirus infection. Under the electron microscope, both cases showed severe loss of myelinated fibers, scanty regenerative clusters and severe damage to the UF population characterized by major axonal loss, numerous empty Schwann cell subunits and, in case 2, numerous minute regenerating axons. They also exhibited abundant formation of collagen pockets and prominent endoneurial fibrosis (Fig. 1).

Fig. 1 Case 1. Electron microscope: numerous collagen pockets (arrowhead) and denervated Schwann cell bands of unmyelinated type (arrow) with prominent endoneurial fibrosis. $\times 7850$

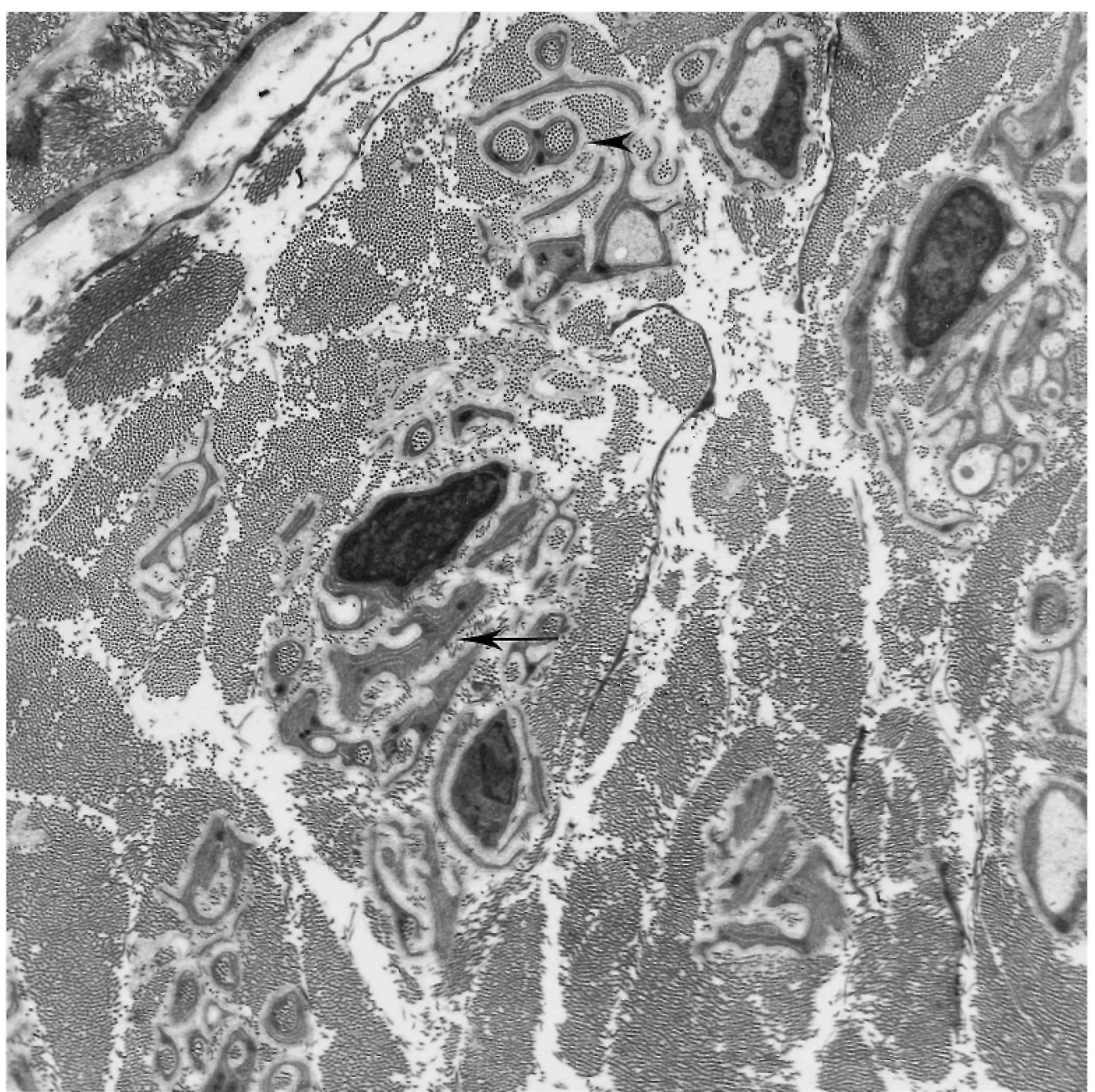




\section{Nerve processing}

Whole nerve biopsy specimens ( $3 \mathrm{~cm}$ long) of the superficial branch of the peroneal nerve were taken at the junction of the lower and middle thirds of the leg [19]. Nerve specimens were gently stretched over blotting paper and fixed in $4 \%$ glutaraldehyde in $0.1 \mathrm{M}$ phosphate buffer at room temperature for $2 \mathrm{~h}$ followed by overnight fixation at $4{ }^{\circ} \mathrm{C}$. The nerves were sectioned into 4-mm lengths. These were then divided to provide pairs of 2-mm-long segments. For each case, one of the paired segment was fixed with osmium tetroxide and routinely processed in epoxy resin for ultrastructural analysis. The other was embedded in epoxy resin without prior osmication; semithin sections were stained by the Bodian-Luxol technique. For each case, the first ultrathin section observed under the electron microscope was the mirror image of the paired semithin section examined under the light microscope after histochemical staining.

\section{Ultrastructural methods to determine the total numbers and densities of UF}

Sections of the entire cross-section of whole peroneal nerves ( $70 \mathrm{~nm}$ thick) were cut with diamond knives, placed on celloidin membranes, mounted on copper hole grids (GA 2000 Agar), and double-stained with uranyl acetate and lead citrate. They were examined under a Philips CM 10 electron microscope at $60 \mathrm{kV}$. For each case, a small fascicle was selected and initially surveyed at a final magnification of $\times 1600$. Then overlapping electron micrographs covering the surface of the whole fascicle were taken at a final magnification of $\times 8625$ and questionable areas were detailed at a final magnification of $\times 14,000$. The three following parameters were assessed:

1. Total number of unmyelinated axons; in accordance with most authors [2-5, 13-15, 18], a first count of unmyelinated axons considered all profiles meeting two or more of the following criteria: (1) rounded profiles surrounded by Schwann cell processes and exhibiting a distinct mesaxon, (2) lighter appearance of axoplasm compared to Schwann cell cytoplasm, (3) larger number of micro-tubules in axoplasm than cytoplasm, and (4) greater electron-density of the axolemma compared to the Schwann cell plasmalemma. Also included in this count were a few unmyelinated axons belonging to Schwann cell complexes of myelinated type, using the criteria proposed by Ochoa and Mair [15]. These values are indicated in Table 1 under the heading 'Total counts'. Two additional subpopulations of axons were obtained by considering only axons of a caliber larger than $0.5 \mu \mathrm{m}$ and axons larger than $0.8 \mu \mathrm{m}$ (Table 1). Densities per $\mathrm{mm}^{2}$ were calculated by dividing the absolute number of unmyelinated axons by the total endoneurial area of each fascicle as measured on semithin sections (see below).

2. Empty Schwann cell subunits; these were identified using the criteria proposed by Ochoa and Mair [15] for typing of Schwann cell complexes.

3. Size-frequency histograms: a minimum of 150 fibers were evaluated, the diameter of each nerve fiber was determined as the mean of the largest diameter and the diameter at right angles to it [3].

\section{Bodian-Luxol staining}

The technique of staining was basically similar to the one routinely used on sections from paraffin blocks. Its application to resin-embedded tissue requires two preliminary steps: the omission of osmium tetroxide post fixation and the removal of superficial resin from the semithin section [10].

\section{Resin removal}

The plastic was removed according to the method of Lane and Europa [9]. In summary, air-dried sections were deplasticized in a saturated solution of $\mathrm{NaOH}$ in absolute ethanol. The saturated solution is matured for 1 week or until it shows a cognac-like color, at which time it is ready for use. Sections were immersed in the solution in a covered jar for $30 \mathrm{~min}$. The saturated solution was removed and sections were washed three times for $10 \mathrm{~min}$ in absolute ethanol.

\section{Bodian 's method}

Sections were rinsed in tap water and placed in a solution of $100 \mathrm{ml} 1 \%$ silver proteinate (Roques-Paris) in distilled water supplemented with $2 \mathrm{ml} 5 \% \mathrm{CuSO}_{4}$ overnight at $37^{\circ} \mathrm{C}$. The copper in the solution impairs nonspecific uptake of silver proteinate by connective tissue. Following development in two sequential reductive solutions of hydroquinone in $\mathrm{Na}_{2} \mathrm{SO}_{3}$ and $\mathrm{Na}_{2} \mathrm{SO}_{4}$, a very fine localization of neurofibrils in axons is achieved. Additional passages through solutions of oxalic acid and gold chloride provided contrast enhancement. Fixation 
was achieved in $\mathrm{Na}_{2} \mathrm{~S}_{2} \mathrm{O}_{3}$.

\section{Luxol counterstain}

Following overnight incubation in $0.1 \%$ Luxol fast blue supplemented with $\mathrm{CH}_{3} \mathrm{COOH}$ at $60{ }^{\circ} \mathrm{C}$, slides were differentiated in $\mathrm{Li}_{2} \mathrm{CO}_{3}$ and $70{ }^{\circ} \mathrm{C}$ methanol, rinsed, dehydrated and mounted with Entellan (Merck, Belgolabo, Overijse, Belgium).

Fig. 2 a-c Control 1. a Photographic reconstruction of a whole nerve fascicle by overlapping micrographs of a semithin section stained by the Bodian-Luxol method. b Optic microscope: unmyelinated axons are identified as dark brown spots clustered around Schwann cell nuclei. c Partial disintegration of myelin sheath in large myelinated fibers (arrowhead) with good preservation in small myelinated fibers (arrow). $a \times 100: b \times 1000 ; c$ $\times 1200$

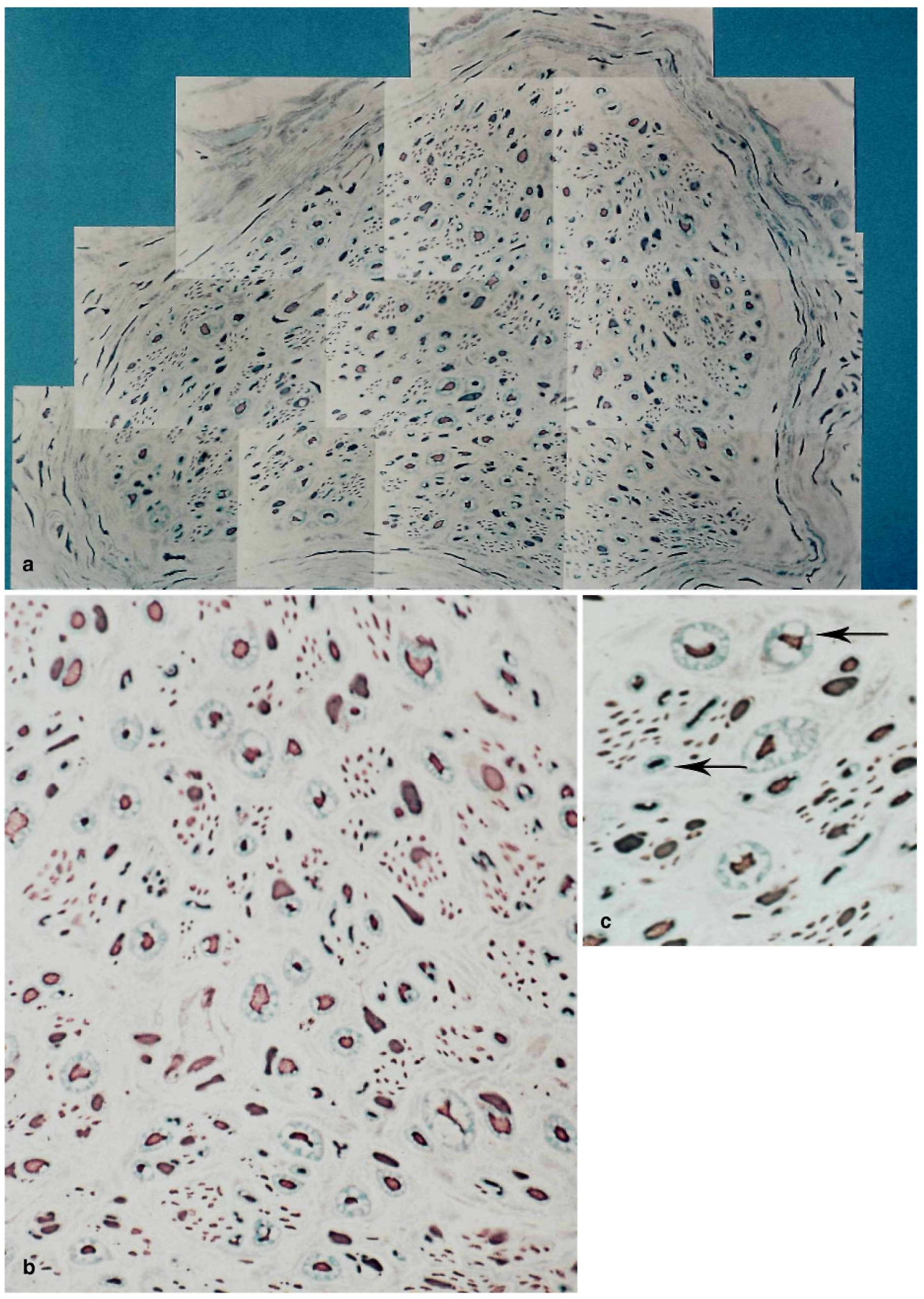




\section{Determination of UF density on Bodian-Luxol preparations}

Overlapping micrographs of each fascicle were taken under oil immersion at an original magnification of $x$ 1000. The whole fascicle was reconstituted at a final magnification of $\times 4000$ (Fig. 2a). Absolute numbers of unmyelinated axons were estimated by manual count by two independent observers on each of the selected fascicles. Total endoneurial surface area of each fascicle was measured by a cell analysis system CAS 200 (Becton Dickinson, San Diego, Calif.). Densities of UF were calculated by dividing the total number of unmyelinated axons by the total endoneurial area of the fascicle.

\section{Determination of myelinated fiber density}

Numbers of myelinated fibers per fascicle were determined by manual counting of both toluidine blue- and Bodian-Luxol-stained semithin sections at optical magnification of $\times 1000$. Densities were calculated as described above.

\section{Statistical methods}

Correlation between optic and ultrastructural counts was assessed by the intra-class correlation coefficient and the lower $5 \%$ confidence limit [1]. The intra-class correlation coefficient ranges from 0 to 1 , with 1 representing perfect correlation between ratings.

\section{RESULTS}

Under the light microscope, on semithin sections stained by the Bodian-Luxol method, axons appeared as small dark brown spots clustered around a Schwann cell nucleus (Fig. 2b). They showed an uneven distribution within the fascicle, being more concentrated at the periphery and more often associated with small-diameter myelinated fibers. The staining of the myelin sheath by Luxol was faint and irregular on large myelinated fibers, probably due to partial myelin disintegration in the absence of prior osmication. Small-diameter myelinated fibers showed a bright blue staining of their myelin sheath allowing for excellent discrimination from large unmyelinated axons (Fig. 2c). A faint background was present in cases 1 and 2 due to nonspecific light-blue staining of abundant endoneurial collagen by Luxol dye, but it did not impinge upon the recognition of unmyelinated axons. Schwann cell nuclei were lightly stained by silver proteinate, but they could be recognized quite easily by their shape and caliber (Fig. 2c). Each fascicle demonstrated a small number of unmyelinated axons cut tangentially that never exceeded $2 \%$ (data not shown). Values of the UF population obtained by light microscopy are indicated in Table 1. Excellent correlation was obtained between observers. Controls showed densities of $44,452 / \mathrm{mm}^{2}$ and $29,571 / \mathrm{mm}^{2}$, respectively, which are within the normal range for the patients' age $[8,12,15,21]$. Values of $13,730 / \mathrm{mm}^{2}$ incase 1 and $1259 / \mathrm{mm}^{2}$ in case 2 indicated pathological axonal loss referring to the same standards.

On semithin sections stained with toluidine blue, control cases showed myelinated fiber densities of $8167 / \mathrm{mm}^{2}$ (control 1) and $8714 / \mathrm{mm}^{2}$ (control 2). Case 1 had a density of $729 / \mathrm{mm}^{3}$, indicating major fiber loss. No residual myelinated fibers were found in the fascicle assessed in case 2. Determination of myelinated fiber density with Bodian-Luxol staining gave consistently lower values due to poor preservation of the myelin sheath of large myelinated axons (data not shown).

Ultrastructurally, control specimens did not show significant postmortem changes probably due to the short interval between death and sampling [8]. Axonal and Schwann cell membranes and organelles were well preserved (Fig. 1 a). No regenerative myelinated type clusters were seen in the fascicles assessed by morphometry. In both controls, fiber-size histograms of UF showed unimodal curves with peaks at $1.2 \mu \mathrm{m}$ (control 1) and $1.3 \mu \mathrm{m}$ (control 2). Unmyelinated axons with diameters larger than $0.8 \mu \mathrm{m}$ represented $65 \%$ (control 1) and 84\% (control 2) of the population of UF [15]. Densities of empty Schwann cell bands of $2129 / \mathrm{mm}^{2}$ (control 1) and 2429/. $\mathrm{mm}^{2}$ (control 2) were within the normal range for the patients' age [8]. Case 1 exhibited a severe loss of myelinated fibers of all caliber and a moderate loss of UF. Massive axonal loss was characteristic of case 2 with complete disappearance of myelinated fibers. In neither case were regenerative myelinated type clusters seen in the fascicle assessed. Both cases showed numerous empty Schwann cell subunits (Fig. 1), but these were particularly abundant in case 2 with a density of 13,666/mm² (Table 1). Collagen pockets were numerous in both cases and endoneurial fibrosis was prominent (Fig. 1). Profiles larger than $0.8 \mu \mathrm{m}$ represented $69 \%$ of unmyelinated axons in case 1 . Case 2 was characterized by a predominance of minute axons with only $21 \%$ of profiles larger than $0.8 \mu \mathrm{m}$. These were distributed in a unimodal fiber-size histogram with a peak value at $0.4 \mu \mathrm{m}$. 
Table 1 Optic and ultrastructural counts of unmyelinated axons. These counts represent absolute numbers of unmyelinated axons in each of the fascicles assessed. Total represents the number of unmyelinated axons considering all profiles; subpopulation of unmyelinated axons with diameter $>0.5 \mu \mathrm{m}$ or $>0.8 \mu \mathrm{m}$ are shown (ESS density of empty Schwann cell subunits)

\begin{tabular}{lcccccc}
\hline Cases & $\begin{array}{c}\text { Optic counts } \\
\text { (Bodian-Luxol) }\end{array}$ & \multicolumn{3}{c}{ Ultrastructural counts } & Endoneurial area & $\begin{array}{c}\text { ESS } \\
\left(/ \mathrm{mm}^{2}\right)\end{array}$ \\
\cline { 2 - 5 } & 1378 & 1871 & 1504 & 1215 & $0.031 \mathrm{~mm}^{2}$ & $2129 / \mathrm{mm}^{2}$ \\
Control 1 & 621 & 726 & 676 & 607 & $0.021 \mathrm{~mm}^{2}$ & $2429 / \mathrm{mm}^{2}$ \\
Control 2 & 508 & 621 & 511 & 425 & $0.037 \mathrm{~mm}^{2}$ & $3135 / \mathrm{mm}^{2}$ \\
Patient 1 & 34 & 179 & 46 & 38 & $0.027 \mathrm{~mm}^{2}$ & $13666 / \mathrm{mm}^{2}$ \\
\hline Patient 2 & & & &
\end{tabular}

Numbers of unmyelinated axons obtained by optic counts were consistently lower than total counts of axonal profiles derived from electron micrographs (Table 1). This result argues against a nonspecific staining of nonaxonal structures such as Schwann cell processes, collagen pockets or endoneurial fibroid tissue. This was obvious in case 2 where only 34 unmyelinated axons were counted at the optic microscope in a fascicle showing a high density of denervated Schwann cell bands and collagen pockets (Fig. 1).

To delineate the sensitivity of the Bodian-Luxol staining, defined as the smallest axonal profile detected, we compared ultrastructural and light microscopic densities of UF. Densities measured by light microscopy correlated best with ultrastructural counts considering all profiles larger than $0.5 \mu \mathrm{m}$, with an intra-class correlation coefficient of 0.992 and a lower $5 \%$ confidence limit of 0.919 . We obtained a slightly lower intraclass correlation coefficient value of 0.987 (lower 5\% confidence limit of 0.893 ) when comparing optical counts and the ultrastructural subpopulation of profiles larger than $0.8 \mu \mathrm{m}$ (Fig. 3). These data suggest that this BodianLuxol technique permits light microscopic detection of axonal profiles as small as $0.6 \mu \mathrm{m}$. The one exception is that of case 2 , where the optical density $\left(1259 / \mathrm{mm}^{2}\right)$ of UF correlated better with ultrastructural count assessing only profiles larger than $0.8 \mu \mathrm{m}\left(1407 / \mathrm{mm}^{2}\right)$. This could indicate decreased sensitivity of the method when dealing with nerves showing prominent endoneurial fibrosis.

Fig. 3 Comparison of optic and ultrastructural densities of unmyelinated nerve fibers. For each case, light microscopy (L.M.) and ultrastructural densities for axons larger than $0.5 \mu \mathrm{m}$ and $0.8 \mu \mathrm{m}$ are plotted together. Excellent correlation is readily apparent

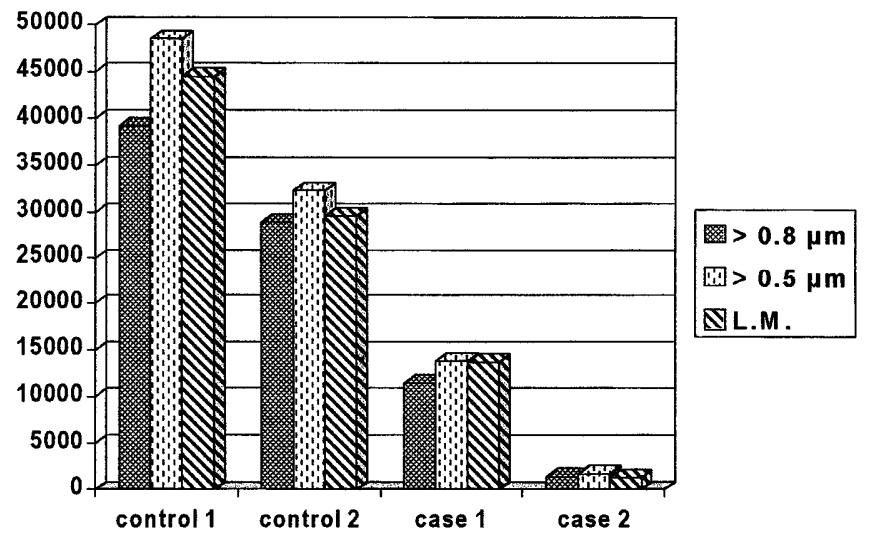

\section{DISCUSSION}

Evaluation of UF in biopsy samples of cutaneous nerves may facilitate the recognition of specific pathological entities. Even though UF are involved in most neuropathies [3, 5, 12, 13, 18, 20], a predominant loss of unmyelinated axons, often associated with selective loss of small-diameter myelinated fibers, has proved a valuable finding with a limited differential diagnosis: leprosy, amyloidosis, small-fiber diabetic neuropathy, and hereditary conditions such as Fabry's disease, Tangier's disease, hereditary sensory and autonomic neuropathies, idiopathic acute pandysautonomia, idiopathic chronic anhydrosis, dimethylaminopropionitrile toxic neuropathy 
and some forms of alcoholic neuropathy $[6,11,15]$.

Direct documentation of neuropathic pain can be achieved only by methods such as microneurography allowing for recording and stimulation of a single nerve fiber, but these techniques are not used routinely. Indirect information can be gained from therapeutic trials, quantitative sensory testing and thermography and histological evidence of nerve pathology. Because of their important content in UF relaying noxious stimulation, cutaneous nerves offer the opportunity to study this component of the primary afferent unit in the nociceptive pathway. Routine evaluation of the population of unmyelinated axons might be of interest in patients with painful cryptogenic chronic neuropathies who are often candidates for peripheral nerve biopsy. Conversely, it might also be of value in patients presenting with selective or predominant loss of pain sensation and/or symptoms or signs of dysautonomia.

Recognition of UF disease is classically based on electron microscopic examination. Ultrastructural quantitative morphometry represents a powerful but time-consuming method. Electron microscopic inspection at low magnification can be useful in the diagnostic workup, but the small size of the sample and the interference of the grid may be a hindrance to quantitative assessment. Alternatively, we propose a histochemical technique based on silver impregnation of axonal neurofibrils on semithin sections. The staining appears specific for axons and does not color Schwann cell profiles or collagen pockets. The sensitivity of the method, defined as the minimal caliber of axon detected, has been estimated at $0.6 \mu \mathrm{m}$ by correlating counts obtained with light and electron microscopy. In the case of massive endoneurial fibrosis, such as in case 2, the smallest axons detected seem to be in a range of 0.7-0.8 $\mu \mathrm{m}$, but are still clearly identifiable. Schwann cell nuclei may show abnormal nonspecific staining, but they are easily recognized by their shape and size. Theoretically, this might limit the value of the technique in cases of giant axon neuropathy.

Quantitation of unmyelinated axons has both practical and theoretical limitations. Distribution of unmyelinated axons is uneven inside a fascicle and a variation up to $29 \%$ between fascicles is currently admitted [2]; thus, the sampling must consider at least four fascicles and a minimal endoneurial area of $0.02 \mathrm{~mm}^{2}$ [4]. Our method allows for an overview of the whole nerve on semithin transverse section at the light microscope; this might obviate the sampling error and help to select specific areas of interest for ultrastructural study. Counts of unmyelinated axons as an index of damage to UF also have theoretical limitations: (1) densities among normal individuals range from 19,000 to $65,000 / \mathrm{mm}^{2}$ for the sural nerve [4]; thus only a massive dropout will reach significance; and (2) given the rapid ability of injured unmyelinated axons to produce sprouts, regeneration is almost unfailing in chronic processes, therefore counts may often provide normal or increased values in case of diseased UF [13-15]. Our method offers a specific staining of axons with diameters larger than $0.6 \mu \mathrm{m}$. Therefore, the population of smallest axons, which have been shown to correspond mainly to regenerative sprouts, is not detected [15]. UF numbers determined by an appropriate silver impregnation technique could allow the detection of axonal loss without adulteration by regenerative axons. Finally, Behse et al. [2] have shown the interest in expressing total number of UF per nerve rather than per $\mathrm{mm}^{2}$ to avoid artefacts due to the variation of endoneurial area. This strategy could be applied more easily at the level of light microscopy.

As an isolated parameter, quantitation is insufficient for evaluating UF disease. Fiber-size histograms as an indicator of regeneration, and quantitation of empty Schwann cell subunits as a gross approximation of axonal loss are mandatory. Ultrastructural morphometry remains the standard method for early detection and exhaustive study of changes in the UF population $[2,8]$. Our method of histochemical staining does not allow measurement of axonal diameter. It does not differentiate UF from those regenerating unmyelinated axons which are to be remyelinated. However, we are confident that this method of Bodian-Luxol staining on semithin sections will prove a valuable tool in routine practice. It offers the light microscopist a reliable means by which to estimate axonal loss among unmyelinated fibers.

\section{Acknowledgements}

We thank Mrs. Michèle Mozin, Mrs. Annette Houbrechts and Mr. Olivier Hougrand for their excellent technical assistance and Mrs. Inge Bats for invaluable photographic aid. We also thank Prof. J.-M. Schröder for helpful comments. This work was partly supported by grants from the University Hospital of Liège and by the Flanders Fund for Scientific Research. 
Published in : Acta Neuropathologica (1999), vol. 98, pp. 323-329.

Status : Postprint (Author's version)

\section{References}

1. Bartko JJ (1966) The intraclass correlation coefficient as a measure of reliability. Psychol Rep 19:3-11

2. Behse F, Buchtal F, Carlsen F, Knappeis GG (1974) Endoneurial space and its constitutents in the sural nerve of patients with neuropathy. Brain 97:773-774

3. Behse F, Buchtal F, Carlsen F, Knappeis GG (1975) Unmyelinated fibers and Schwann cells of sural nerve in neuropathy. Brain 98:493510

4. Gibbels E (1989) Morphometry of unmyelinated fibers. Clin Neuropathol 8:179-184

5. Gibbels E, Kentenich M (1990) Unmyelinated fibers in sural nerve biopsies of chronic inflammatory demyelinating polyneuropathy. Acta Neuropathol 80:439-447

6. Hanyu N, Ikeda S, Nakadai A, Yanagisawa N, Powell HC (1989) Peripheral nerve pathological findings in familial amyloid polyneuropathy: a correlative study of proximal sciatic nerve and sural nerve lesions. Ann Neurol 25:340-350

7. Johnson PC, Beggs JL, Olafsen AG, Watkins CJ (1994) Unmyelinated nerve fiber estimation by immunocytochemistry. Correlation with electron microscopy. J Neuropathol Exp Neurol 53:176-183

8. Kanda T, Tsukagoshi H, Oda M, Miyamoto K, Tanabe H (1991) Morphological changes in unmyelinated nerve fibres in the sural nerve with age. Brain 114:585-599

9. Lane BP, Europa DL (1965) Differential staining of ultrathin section of Epon-embedded tissues for light microscopy. J Histochem Cytochem 13:579

10. Larsson L-I (1986) Plastic embedding technique. In: Larsson L-I (ed) Immunocytochemistry: theory and practice. CRC Press, Boca Raton, pp 61-67

11. Llewelyn JG, Gilbey SG, Thomas PK, King RHM, Muddle JR, Watkins PJ (1991) Sural nerve morphometry in diabetic autonomic and painful sensory neuropathy. Brain 114: 867-892

12. Midroni G, Bilbao JM (1995) Quantitative technique. In: Midroni G, Bilbao JM (eds) Biopsy diagnosis of peripheral neuropathy. Butterworth-Heineman, Newton, pp 38-40

13. Ochoa J (1978) Recognition of unmyelinated fiber disease: morphologic criteria. Muscle Nerve 1:375-387

14. Ochoa J, Mair WG (1969) The normal sural nerve in man. I. Ultrastructure and numbers of fibres and cells. Acta Neu- ropathol (Berl) $13: 197-216$

15. Ochoa J, Mair WG (1969) The normal sural nerve in man. II. Changes in the axons and Schwann cells due to ageing. Acta Neuropathol (Berl) 13:217-239

16. Ranson SW (1911) Non-medullated nerve fibres in the spinal nerves. Am J Anat 12: 67-87

17. Reznik M (1996) Structure et fonctions du système nerveux cutané. Pathol Biol (Paris) 44: 831-837

18. Schroder JM, Gibbels E (1977) Marklose Nervenfasern im Senium und im Spätstadium der Thalidomid-Polyneuropathie: quantitativelektronmikroskopische Untersuchungen. Acta Neuropathol (Berl) 39:271-280

19. Stevens JC, Lofgren EP, Dyck PJ (1973) Histometric evaluation of branches of peroneal nerve: technique for combined biopsy of muscle nerve and cutaneous tissue. Brain Res 52: 37-59

20. Thomas PK, Ochoa J (1993) Clinical features and differential diagnosis. In: Dyck PJ, Thomas PK (eds) Peripheral neuropathy. Saunders, Philadelphia, pp 757-764

21. Thomas PK, Berthold C-H, Ochoa J (1993) Microscopic anatomy of the peripheral nervous system: nerve trunks and spinal roots. In: Dyck PJ, Thomas PK (eds) Peripheral neuropathy. Saunders, Philadelphia, pp 56-66, 71-72 